\title{
Rapid tips to improve the quality of science
}

\section{Leonardo Bianco de Carvalho}

There are many reports, papers, websites, videos, and other communications showing the importance of the quality of writing to publish the results of research projects in high-quality journals. However, the quality of science is not only dependent on the quality of writing. Actually, the quality of writing papers is the final issue researchers should be worried about (but it is obviously not less important). Initially, the first step to propose and carry out a relevant research, which is the basis for good science, is dependent on the capacity of understanding what is needed for making good science.

The first tip to make good science is easy to know. Researchers should know what not good science is. On the other words, they must be sure what the difference between good science and bad science is! BELUZ (2013) listed six red flags to watch for that tell the difference. Here, I have summarized and proposed four questions to be answered:

1) Is the sample and the condition representative?

You have to keep in mind the population (sampling) that is studied is not always able to be generalized, despite how it is framed by the writing about the study. In accordance with the idea of BELUZ (2013), findings in greenhouse conditions, for example, might suggest similar response under field conditions, but they must be proved. As well, the sample population or condition might not be representative in other ways. So, any extrapolation on effects under field conditions should be read with a critical gaze.

2) How would this study square in the real world?

The second question is on whether the treatment that the study looked at is consistent with the way it would exist in the real world (BELUZ 2013). If researchers test just a half-field-dose of a pesticide and the pest is not controlled, they cannot affirm that the pest is resistant. Another example of BELUZ (2013) refers to studies of pharmaceuticals, where drugs are often compared with a placebo to establish that they work, but that is not necessarily a fair comparison when there are other similar treatments available and clinicians and patients want to know whether the new drug is better than the best-available drug.
3) How big is the study?

Larger studies involving more people at different sites are generally better than smaller studies (BELUZ 2013). That is because big studies are usually more representative of the broader population and less influenced by extreme cases than small studies. Other point is that larger studies generally involve different research teams with high skill levels, knowledge, background, and wisdom (as a consequence). So, the quality of these studies is probably not questioned.

4) What about the other evidence?

BELUZ (2013) explains that single studies are not the best way to tell you about what is really happening. In fact, according to this author, this kind of reporting is probably misrepresentative and misleading, A single study will not change (and never should) the view the problem, but many studies, in different contexts, using different methods, on different populations, will (BELUZ 2013).

The second tip to make good science is simple to understand and is related to the diversity in the research team. According to Shankar Vendatam, as science becomes more diverse, scientific collaborators are growing more diverse too (NPR 2014). As a consequence, the concepts, ideas, views, experiences, and much more, related to the research team, are all diverse. Due to this point, working in a group, especially in research teams with high diversity, allows for identifying a good scientific and relevant problem to study as well as permitting the creation of highly significant hypothesis to be tested. According to Richard Freeman, ethnic diversity is an indication of an ideas' diversity; people who are more alike are likely to think more alike and one of the things that gives a kick to science is that you get people with somewhat different views (NPR 2014). So, avoiding group thinking is essentially good for science, concluded David Greene (NPR 2014).

The third tip is on choosing a good scientific problem. So, what is a good scientific problem? According to ALON (2009), a good choice means that researchers can competently discover new knowledge that they find fascinating and that allows self-expression. But, how do researchers choose one?

São Paulo State University, Dracena, SP, Brazil<lbcarvalho@dracena.unesp.br> 
The answer is not simple for everyone. ALON (2009) proposes that the starting point has to aim to choose a problem for scientists in order to foster growth and self-motivated research. A second point is related to the skills of the researchers and the technology of the lab, and also related to the interest in increasing the knowledge expected from the project, as describes ALON (2009). Obviously, many labs can find big scientific problems that the technology of their labs does not allow solving them; or that even the skills of the research team are not sufficient to permit finding the solutions. Thus, the choice of a good scientific problem must be based firstly on the real capacity of both research teams and labs.

Another point, according to ALON (2009), is that researchers have to consider both feasible and high interest scientific problems, likely to extend their knowledge significantly. However, this combination is also not easy to find and should be considered as a function of the life stages of the scientist. For example, a beginning graduate student needs an easy problem while a postdoc student would prefer a hard one. In any cases, feasible problems must also be interesting problems, in accordance obviously to the reality of the research team and lab. But how do researchers identify an interesting scientific problem? The answer... reading, studying, searching for general information, thinking and thinking over... discussing and reading again... and having the feeling for science! In addition, ALON (2009) proposes that when researchers consider listening to their inner voice, they become more likely to choose problems that will satisfy them in the long term. This author also emphasizes that the inner voice can be strengthened and guided if one is lucky enough to have caring mentors.

Well... but what is the essence of the inner voice? The projects that a particular researcher finds interesting are an expression of a personal filter, a way of perceiving the world (ALON 2009). This author explains that this filter is associated with a set of values: the beliefs of what is good, beautiful, and true versus what is bad, ugly, and false; our unique filter is what we bring to the table as scientists; a multiplicity of styles and questions, based on the uniqueness of scientists, is the basis of viable and creative science. To choose a good problem, therefore, researchers need to reflect on their own world view to have the potential to achieve self-expression in science; so, when one can achieve self-expression in science, work becomes revitalizing, self-driven, and laden with personal meaning, so that it may also have a better chance of discovering something profound (ALON 2009).

The fourth tip is to look for a mechanism not for a phenomenon. HENDRIX (2015) explains that a very common mistake young researcher's do is to fall in love with descriptive analyses. Researchers can spend many years just precisely describing correlations, showing fancy images of receptor expressions or dramatic morphological or biochemical changes in test and control tissues. However, whenever researchers find a causal link between two effects the quality of their study will increase, explains that author. Thus, look for a functional test which demonstrates that the effect one describes can be significantly increased or reduced by a well-defined intervention (HENDRIX 2015).

Thus, researchers will just have a chance to publish a high impact paper if they have significant and relevant conclusions to be published. In the same way, researchers will have good conclusions just if they have a good problem to solve. The identification and the choice of a problem to be studied is the first step of planning a good research project. Thus, the quality of science and paper publication starts with the good ideas researchers have, not just on the time of writing.

\section{SOURCES}

ALON U. 2009. How to choose a good scientific problem. Available at $<\mathrm{http}$ ://www.imbb.forth.gr/people/aeconomou/ pdf/HowToChooseGoodProblem.pdf $>$. Accessed on May 23, 2016.

BELUZ J. 2013. Good science vs. bad science. Available at: $\quad<$ http://www.macleans.ca/authors/julia-belluz/goodscience-vs-bad-science/>. Accessed on: May 23, 2016.

HENDRIX S. 2015. 10 simple strategies to increase the impact factor of your publication. Available at: $<$ http:// www.smartsciencecareer.com/increase-the-impact-factorof-your-publication/>. Accessed on: May 23, 2016.

NPR - NATIONAL PUBLIC RADIO. 2014. Does Diversity On Research Team Improve Quality Of Science? Interview. Available at: <http://www.npr.org/2014/03/21/292225798/ does-diversity-on-research-team-improve-quality-ofscience>. Accessed on: May 23, 2016. 